\title{
Dental Caries Experience, Oral Hygiene Status, and Traumatic Dental Injuries among the Visually Impaired in Jabalpur, Madhya Pradesh
}

\author{
Vinay Suresan
}

\begin{abstract}
Aim and objective: To assess the dental caries experience, oral hygiene status, and traumatic dental injuries (TDI) among the visually impaired in Jabalpur, Madhya Pradesh, India.

Materials and methods: A descriptive cross-sectional study was conducted among 318 subjects. The questionnaire recorded the DecayedMissing-Filled Tooth Index (DMFT), Oral Hygiene Index-Simplified [OHI(S)], and TDI. Discrete data were analyzed using Student's $t$-test and continuous data was analyzed using analysis of variance (ANOVA). A $p$ value was set at 0.05 as significant.

Results: The mean age of the study population was $13.9 \pm 3.5$ years. The caries prevalence was $69.2 \%$ with a caries experience of $1.23 \pm 2.16$. The $\mathrm{OHI}(\mathrm{S})$ score was $1.6 \pm 1.0$ and the prevalence of TDI was $27.6 \%$ in this population.

Conclusion:Visually impaired subjects showed a higher dental caries experience, fair oral hygiene status, and extensive TDI. This study population lacked adequate knowledge concerning appropriate usage of oral hygiene aids, accurate brushing techniques, ill-effects of cariogenic diet, and lacked periodic dental visits.

Clinical significance: Children and adults living with disabilities have fewer healthy teeth, more untreated tooth caries, and periodontal diseases compared to the general population. Yet, they have the same rights to good oral health and oral healthcare as the rest of the population. If the dental care needs of these populations have to be met then, there is a need for a refurbished dental curriculum emphasizing the training of the dental team to meet the needs of people with special needs.
\end{abstract}

Keywords: Comprehensive dental care, Decayed-Missing-Filled Tooth Index, Oral hygiene index, Tooth injuries, Visually impaired person.

World Journal of Dentistry (2021): 10.5005/jp-journals-10015-1791

\section{INTRODUCTION}

Worldwide, no less than 2.2 billion people are visually impaired, of whom no less than half have this condition which could have been prevented. ${ }^{1}$ The estimated worldwide prevalence of moderate and severe bilateral vision impairment was 216.6 million people. ${ }^{2}$ Three Asian regions alone account for $62 \%$ of this prevalence, India contributes over 15 million cases alone for the South-East Asia region. ${ }^{3}$ The etiology for visual impairment varies between the developing and developed world. Untreated cataracts caused most of the visual impairment in the developing world. ${ }^{4}$ Whereas agerelated macular degeneration was responsible for visual impairment in developed countries. ${ }^{1}$

It has been established that visual impairment increases the risk of developing dental diseases and silently contributes to poor oral health. ${ }^{5}$ In India, the oral health status of the visually impaired is inferior to their normal counterparts; demonstrating a glaring degree of oral health disparities. ${ }^{6}$ The most common oral characteristics among these individuals are higher dental caries experience and almost always accompanied with inadequate oral hygiene. ${ }^{7}$

Oral health contributes toward an individual's well-being by improving their quality of life. ${ }^{8}$ It is recognized that the visually impaired fail to sense and distinguish the initial signs and symptoms of oral diseases and take appropriate remedial measures because of their impaired sensory, motor, and intellectual incapacities. ${ }^{9,10}$ Visually impaired subjects are highly susceptible to trauma while they are acquiring motor skills. As a consequence trauma to the
Pacific Academy of Higher Education and Research University, Udaipur, Rajasthan, India

Corresponding Author: Vinay Suresan, Pacific Academy of Higher Education and Research University, Udaipur, Rajasthan, India, Phone: +918763799695, e-mail: reachvnys@gmail.com

How to cite this article: Suresan V. Dental Caries Experience, Oral Hygiene Status, and Traumatic Dental Injuries among the Visually Impaired in Jabalpur, Madhya Pradesh. World J Dent 2021;12(1):50-56. Source of support: Nil

Conflict of interest: None

anterior teeth leading to hypoplastic teeth occurs with greater incidence than their normal peers. ${ }^{11}$

Oral health education is a key to lessen the risk of dental caries and periodontal diseases. Conventional methods of oral health education involving the use of audio-visual aids, visual cues that disclose plaque holds obsolete as it is based completely on the sense of vision which is lacking in these individuals. ${ }^{12}$ Children and adults living with disabilities have the same rights to good oral health and oral healthcare as the rest of the population. Regrettably, this is the utmost neglected health needs among this population. ${ }^{13}$

Regardless of the study claiming a high prevalence of visually impaired in central India, ${ }^{14}$ data are scarce in regard to the oral disease burden of visually impaired in Jabalpur city. Hence, the present study aimed to assess the dental caries experience, oral hygiene status, and traumatic dental injuries (TDI) among the 
visually impaired in Jabalpur, Madhya Pradesh, India. The study had the following objectives: to compare the dental caries experience with sugar consumption habits, oral hygiene index scores to their oral hygiene practices, and the provision of comprehensive dental care protocol (CDCP).

\section{Materials and Methods Informed Consent/Ethical Considerations}

Permission to conduct the survey and the treatment procedures for the visually impaired under both government and private organizations in Jabalpur city was obtained from the Department of Disabilities and Social Empowerment, Government of Madhya Pradesh. The school teachers/wardens were used as guardians for obtaining consent for minors after explaining the purpose and the need for the study. The participants' identity was kept confidential. The institutional ethical review committee approved the study.

\section{Study Design}

This study followed a descriptive cross-sectional study design. The dental caries experience, oral hygiene status, and TDI were assessed among the visually impaired in Jabalpur, Madhya Pradesh. This study was conducted and reported in accordance with the Strengthening the Reporting of Observational Studies in Epidemiology (STROBE) Statement: guidelines for reporting observational studies.

\section{Setting and Participants}

This oral healthcare program was conducted for 6-30 years' old visually impaired subjects of Jabalpur, Madhya Pradesh, India. The subjects were selected from a list comprising of four schools and hostels (one under the district administration and the remaining three managed by private organizations). The administrative heads of these schools were approached and permission was sought after explaining the purpose and nature of the survey.

\section{Study Size}

The sample size estimation was done using the empirical data of studies among the visually impaired. The sample size adequate for this study was derived after substituting; a disease prevalence of $49 \%$, confidence interval of $95 \%$, and a margin of error set at $5 \%$. An aggregate of 384 subjects made up the sample. A universal sampling technique was employed in this study to allow all the subjects to reap the benefits of this program. On completion of the study, 318 subjects were examined. Coverage of $84.5 \%$ was recorded for this study. The common reason to evade participation was absenteeism during the time of examination followed by non-consenting.

\section{Data Sources/Measurement}

The investigator extracted the details orally from the participants using a 15-item questionnaire which was designed to gather the sociodemographic characteristics, their performance of oral hygiene (material usage, method of brushing, frequency of brushing and changing the toothbrush), and dietary sugar consumption patterns (frequency of intake, time of intake, form, and consistency).

Each subject received a visual examination in accordance with American Dental Association (ADA) specification type III for an oral examination to record Decayed-Missing-Filled Tooth Index (DMFT/dmft), ${ }^{15}$ Oral Hygiene Index-Simplified (OHI(S)), ${ }^{16}$ and TDI. ${ }^{17}$ Oral examinations were carried out by seating the subjects on an ordinary chair and examining them under adequate natural illumination. The recording assistant was close to the examiner aiding in the correct entry of the codes.

\section{Control of Bias}

A single examiner (VS) conducted the oral examinations. Not more than 25-30 subjects were examined every day. The investigator and recording clerk carried out oral examinations on a sample of 30 patients with varying clinical features before the start of the study. These examinations were repeated after 48 hours on the same patients in random order. The examiner demonstrated intraexaminer reliability $\kappa$ value of 0.91 indicating very high reliability.

\section{Comprehensive Dental Care Protocol}

The CDCP adopted in this study was based on proposals for planning and implementation of dental care developed by Cavalcanti et al. ${ }^{18}$ The CDCP was developed to expand access, solve urgencies, diagnose dental needs, control pain and infection, perform restorative treatments, conduct health education, apply preventivepromotional measures, eliminate health needs, conclude treatments, and achieve health promotion. In this protocol, each participant received: diagnosis of oral health needs; referral and resolution of emergencies; curative interventions; application of preventive measures; evaluation; and periodical control through outreach programs conducted in their hostel premises using mobile dental unit and referral to hospital for specialized care.

\section{Statistical Methods}

Data were imported to SPSS Version 18.0. Continuous measurements were presented as mean \pm standard deviation and categorical measurements were presented in numbers (\%). Discrete data were analyzed using Student's $t$-test and continuous data were analyzed using analysis of variance (ANOVA). Post hoc test was applied for situations in which already a significant omnibus $F$ test. Comparison between proportions was done using the Chi-square test. The outcome measures were assessed among the following age groups: $6-12,13-19$, and 20-30 years, gender, and type of schools (government or private). A binary grading system categorizing subjects as "DMFT $=0$ " or “DMFT $\geq$ 1 " for estimation of the association between the caries experience and the sugar consumption was done. A $p$ value was set at 0.05 as significant.

\section{Results \\ Demographic Profile}

The age range of the subjects was $6-30$ years with a mean age of $13.9 \pm 3.5$ years. Among the 318 subjects, 162 (50.9\%) were males and $156(49.1 \%)$ were females. The study population was divided based on the administration of the schools, 163 (51\%) of subjects were in government schools and 155 (49.8\%) of subjects were in private schools (Table 1).

\section{Dental Caries Experience}

The caries prevalence of the study population was $69.2 \%$. Mean DMFT was $1.23 \pm 2.16$ for permanent teeth and mean dmft $0.1 \pm$ 0.46 for primary dentition (age $\leq 12$ years, $n=137$ ). The decayed (D,d) component was the chief contributor to the DMFT score (Fig. 1). Insignificant difference was found while comparing DMFT/dmft with the type of schools and age groups but, a highly significant difference ( $p=0.000$ ) was observed comparing between gender (Table 2). After dichotomizing dental caries as present or absent, 
Oral Health Status of Visually Impaired in Jabalpur, Madhya Pradesh

Table 1: Distribution of the study population according to the school type, gender, and age

\begin{tabular}{lllllc}
\hline \multirow{2}{*}{ School type } & & \multicolumn{4}{c}{ Age in years, $n(\%)$} \\
\cline { 3 - 5 } Govt & Gender & $6-12$ & $13-19$ & $20-30$ & Total \\
& Male & $22(36.1)$ & $42(59.2)$ & $17(54.8)$ & $81(49.7)$ \\
& Female & $39(63.9)$ & $29(40.8)$ & $14(45.2)$ & $82(50.3)$ \\
Private & Total & $61(100.0)$ & $71(100.0)$ & $31(100.0)$ & $163(100.0)$ \\
& Male & $33(43.4)$ & $45(70.3)$ & $3(20.0)$ & $81(52.3)$ \\
& Female & $43(56.6)$ & $19(29.7)$ & $12(80.0)$ & $74(47.7)$ \\
Total & Total & $76(100.0)$ & $64(100.0)$ & $15(100.0)$ & $155(100.0)$ \\
& Male & $55(40.1)$ & $87(64.4)$ & $20(43.5)$ & $162(50.9)$ \\
& Female & $82(59.9)$ & $48(35.6)$ & $26(56.5)$ & $156(49.1)$ \\
& Total & $137(100.0)$ & $135(100.0)$ & $46(100.0)$ & $318(100.0)$ \\
\hline
\end{tabular}

Table 2: Comparisons between the demographics and the caries experience of the subjects

\begin{tabular}{lllllll}
\hline \multirow{2}{*}{ Variables } & \multicolumn{5}{c}{ Caries experience } & \\
\cline { 3 - 6 } Age in years & $D$ & $M$ & $F$ & DMFT & Test \\
& $6-12$ & $1.16 \pm 2.19$ & 0 & 0 & $1.13 \pm 2.20$ & $F=1.344, p=0.262$ \\
& $13-19$ & $1.14 \pm 2.11$ & 0 & $0.01 \pm 0.12$ & $1.05 \pm 2.09$ & \\
Gender & $20-30$ & $1.58 \pm 2.20$ & $0.06 \pm 0.32$ & $0.02 \pm 0.14$ & $1.65 \pm 2.23$ & \\
& Male & $0.73 \pm 1.51$ & $0.01 \pm 0.17$ & $0.01 \pm 0.13$ & $0.67 \pm 1.49$ & $t$ test $=-4.250, p=0.000^{\mathrm{a}}$ \\
School & Female & $1.71 \pm 2.57$ & 0 & 0 & $1.69 \pm 2.59$ & \\
& Government & $1.13 \pm 2.04$ & $0.01 \pm 0.17$ & $0.01 \pm 0.11$ & $1.12 \pm 2.05$ & $t$ test $=-0.450, p=0.653$ \\
\hline
\end{tabular}

${ }^{\text {aStatistically highly significant }(p \leq 0.00)}$

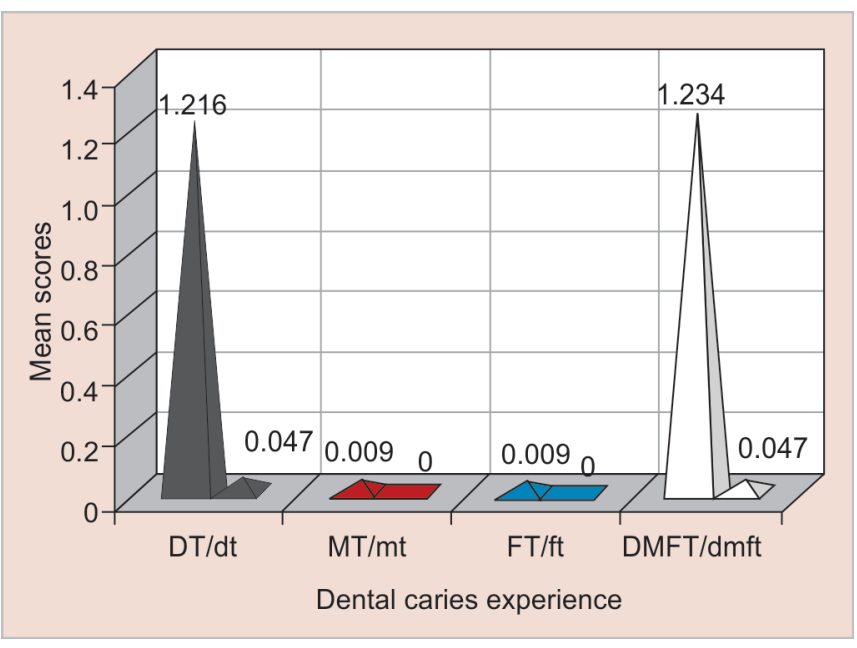

Fig. 1: Dental caries experience of the study population

comparisons were made between the prevalence of caries and the history of sugar consumption, a statistical significance $(p=0.003)$ was observed with the frequency of sugar intake; nearly half of the study population consumed sugary foods at least once daily, the form of sugar being; solid ( $p=0.019)$ and consistency being; sticky $(p=0.004)$ was more cariogenic (Table 3).

\section{Oral Hygiene Status}

The $\mathrm{OHI}(\mathrm{S})$ score was $1.6 \pm 1.0$ for the entire sample which; inferred a fair status. Among the study population, 47, 40, and $13 \%$ revealed fair, good, and poor oral hygiene status, respectively. Demographic characteristics were compared with the OHI(S) scores as presented in Figure 2. The OHI(S) scores recorded across the age groups and school types were statistically insignificant $(p>0.05)$, whereas the $\mathrm{OHI}(\mathrm{S})$ scores between gender was statistically highly significant ( $p=0.00$ ). Table 4 describes the comparison of oral hygiene practices with the mean $\mathrm{OHI}(\mathrm{S})$ scores of the subjects. Bulk of the study population used toothbrush $(n=312,98 \%)$ and toothpaste ( $n$ $=225,80 \%)$ to clean their teeth. The majority brushed their teeth once a day $(n=235,74 \%)$. These variables showed a statistically insignificant $(p>0.05)$ relation with the OHI(S) scores. However, the method of cleaning and frequency of changing the toothbrush was statistically significant ( $p \leq 0.05$ ). Post hoc multiple comparisons when applied ranked vertical < horizontal < circular in the order of their mean OHI(S) scores. Similarly, multiple comparisons when applied on the frequency of changing the toothbrush, 0-3 months was ranked higher than all the other permutations reporting the least $\mathrm{OHI}(\mathrm{S})$ scores.

\section{Traumatic Dental Injuries}

The prevalence of TDI in this study population was $27.6 \%$. Frequency percentages of TDI were as follows: TDI (code 1): $n=16$ (19\%), TDI (code 2): $n=37$ (43\%), TDI (code 3): $n=18$ (21\%), TDI (code 4): $n=8$ (9\%), and TDI (code 5): $n=7(8 \%)$. The most common teeth involved were the permanent maxillary central incisors $n=72$ (83\%).

\section{Comprehensive Dental Care Protocol}

During the study period, 640 work hours were spent, over 160 , 4-hour shifts. During that period, 76 dental outreach visits were carried out, with 318 initial consultations. A total of 570 basic care oral health procedures were performed, of which 302 (52.98\%) were preventive and promotional, while 268 (47.01\%) were curative 
Table 3: Comparison of the prevalence of caries and the history of sugar consumption

\begin{tabular}{|c|c|c|c|c|c|}
\hline \multirow[b]{2}{*}{ Variables } & & \multicolumn{3}{|c|}{ Binary grading } & \multirow[b]{2}{*}{$p$ value } \\
\hline & & $D M F T=0$ & $D M F T \geq 1$ & $\chi^{2}$ & \\
\hline \multirow[t]{4}{*}{ Frequency of sugar intake } & Once & $125(56.8)$ & $55(56.1)$ & 11.454 & $0.003^{a}$ \\
\hline & Twice & $95(43.2)$ & $38(38.8)$ & & \\
\hline & Thrice & 0 & $5(5.1)$ & & \\
\hline & >Thrice & 0 & 0 & & \\
\hline \multirow[t]{2}{*}{ Time of intake } & With meal & $80(36.4)$ & $35(35.7)$ & 0.012 & 0.911 \\
\hline & In between & $140(63.6)$ & $63(64.3)$ & & \\
\hline \multirow[t]{2}{*}{ Form } & Solid & $200(90.9)$ & $80(81.6)$ & 5.545 & $0.019^{a}$ \\
\hline & Liquid & $20(9.1)$ & $18(18.4)$ & & \\
\hline \multirow[t]{2}{*}{ Consistency } & Sticky & $202(91.8)$ & $79(80.6)$ & 8.281 & $0.004^{a}$ \\
\hline & Non-sticky & $18(8.2)$ & $19(19.4)$ & & \\
\hline
\end{tabular}

${ }^{\text {aStatistically highly significant }(p \leq 0.05)}$

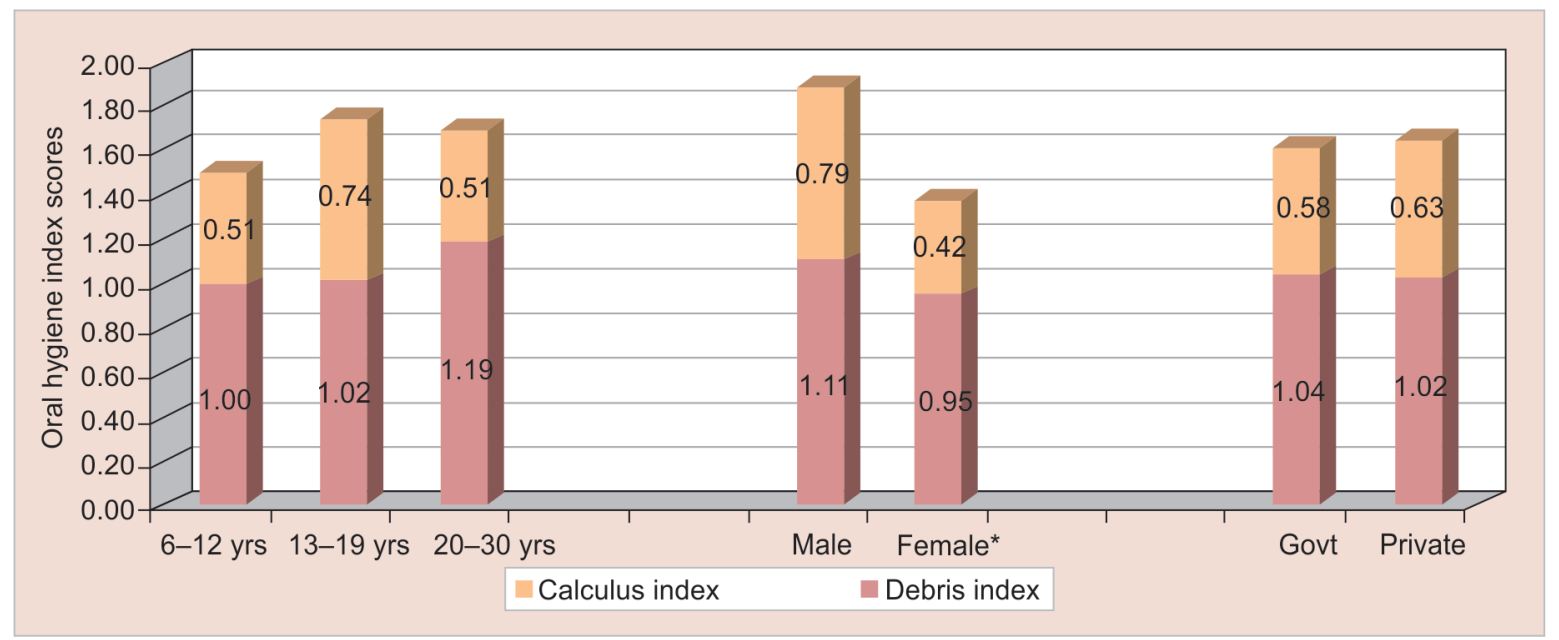

Fig. 2: Comparison of the demographic characteristics of the study subjects and the OHI-S scores

Table 4: Comparison of oral hygiene practices with the mean $\mathrm{OHI}(\mathrm{S})$ scores

\begin{tabular}{|c|c|c|c|c|}
\hline Variables & & $n(\%)$ & $\mathrm{OHI}(S)$ scores (mean $\pm S D)$ & pvalue \\
\hline \multirow[t]{2}{*}{ Type of cleaning } & Tooth brush & $312(98)$ & $1.62 \pm 1.05$ & $t$ test $=-1.510, p=0.190$ \\
\hline & Finger & $6(0.01)$ & $2.40 \pm 1.25$ & \\
\hline \multirow[t]{2}{*}{ Materials used } & Toothpowder & $63(19)$ & $1.59 \pm 0.83$ & $t$ test $=0.374, p=0.709$ \\
\hline & Toothpaste & $255(80)$ & $1.64 \pm 1.11$ & \\
\hline \multirow[t]{3}{*}{ Method of cleaning } & Horizontal & $195(61)$ & $1.60 \pm 1.00$ & $F=4.214, p=0.016^{a}$ \\
\hline & Vertical & $41(13)$ & $1.32 \pm 1.12$ & \\
\hline & Circular & $82(26)$ & $1.88 \pm 1.11$ & \\
\hline \multirow[t]{3}{*}{ Frequency of brushing } & Once & $235(74)$ & $1.56 \pm 1.04$ & $F=1.996, p=0.138$ \\
\hline & Twice & $80(25)$ & $1.88 \pm 1.08$ & \\
\hline & Thrice & $3(0.009)$ & $0.50 \pm 0.00$ & \\
\hline \multirow[t]{4}{*}{ Frequency of changing toothbrush } & $0-3$ months & $156(49)$ & $1.79 \pm 1.11$ & $F=2.589, p=0.053^{\mathrm{a}}$ \\
\hline & 4-6 months & $121(38)$ & $1.51 \pm 1.01$ & \\
\hline & $7-12$ months & $29(9)$ & $1.33 \pm 0.90$ & \\
\hline & 13-24 months & $12(0.03)$ & $1.51 \pm 0.83$ & \\
\hline
\end{tabular}

${ }^{\text {aStatistically significant }(p \leq 0.05)}$

and restorative. Referrals to the specialized care in the hospital, consisted of X-ray requests $(n=87)$, endodontic treatments $(n=76)$, and denture prosthesis $(n=17)$.

\section{Inference}

The study aimed to assess the caries experience, oral hygiene status, and prevalence of TDI along with the objectives of assessing their 
oral hygiene practices and sugar consumption practice. The study population showed caries prevalence in more than half of the subjects. The decayed $(D, d)$ component being the chief contributor to the mean DMFT/dmft. The majority of the subjects brushed their teeth once a day with a fair oral hygiene status. The prevalence of TDI was quite common in the study population indicating susceptibility to trauma to their anterior teeth.

\section{Discussion}

Visual impairment in central rural India was $17 \%$ in the adult population. ${ }^{19}$ Owing to such high prevalence, this was the first comprehensive oral health program conducted to assess oral health status, compare the caries experience with sugar consumption habits, oral hygiene status to their oral hygiene practices, and provision of comprehensive oral healthcare services for the visually impaired aged 6-30 years living in Jabalpur, Madhya Pradesh, Central India.

Oral hygiene habits are acquired early in life and carried on to adulthood. Hence, the need arises for watchful adult supervision during the performance of plaque control measures. Rather, it was found that the visually impaired seldom receive adult supervision hence, were less informed. ${ }^{20}$ This finding is validated by numerous studies reporting deprived oral health status of the visually impaired when matched to their normal counterparts. ${ }^{10,21}$ Many explanations have been put forth to reason out this discrepancy like; the caretakers tend to neglect the oral health of the visually impaired as their primary focus is on managing their existing disability ${ }^{22}$ and visually impaired also has psychological repercussions like sadness, anxiety, and depression owing to the neglect of oral health. ${ }^{23}$

\section{Dental Caries Experience}

The current study reported a dental caries prevalence of $69.2 \%$. Various studies conducted on similar objectives have presented with percentages at par if not higher to the present study: South India $^{10}(40 \%)$, Eastern Odisha, India ${ }^{24}(46 \%)$, Khartoum State, Sudan ${ }^{25}$ (46.8\%), Uttarakhand, India ${ }^{26}$ (57.7\%), Chennai, India ${ }^{27}$ (90\%), and Istanbul, Turkey ${ }^{28}(73.6 \%)$. In the present study, $26.4 \%$ of subjects were caries-free. The higher prevalence of dental caries among the visually impaired reveals a low level of oral health awareness, which was also observed by Chang and Shih. ${ }^{20}$ Many factors contribute toward such high prevalence of dental caries, such as, inability to visually appreciate their oral health, deficient manual dexterity, little importance given to his/her facial appearance, ${ }^{9}$ lack of supervision or assistance provided by the caregivers during toothbrushing, ${ }^{25}$ unfamiliarity with the dental setting, ${ }^{21}$ and the dearth of oral educational programs targeting populations with disabilities and their caregivers. ${ }^{11}$

A few justifications for this can be lack of motivation, poor socioeconomic status, cost of treatment, and available oral healthcare facilities. These deficiencies are the main reasons that the $D(1.21)$ component contributes to the greater part of the DMFT score (1.23). On further exploration, sugar consumption history revealed significant tendencies toward having dental caries (DMFT >1) as the frequency of sugar consumption increases, intake of solid slowly dissolving sugar, and sugars having a sticky consistency. These outcomes are comparable to the study by Shetty et al. ${ }^{29}$ who identified, in-between meal serving of sweet dish as a cause of higher tooth decayed. In the current study, the female gender showed a significantly higher caries prevalence ratio when compared to males.

\section{Oral Hygiene Status}

The inevitable consequences of plaque accumulation can be evaded only through, daily effective oral hygiene measures. Prior studies have proved that the visually impaired have poorer oral hygiene status compared to their normal peers. ${ }^{10,21}$ The present study reports an $\mathrm{OHI}(\mathrm{S})$ score of $1.6 \pm 1.0$ which was inferred as a fair oral hygiene status. The oral hygiene status of $47 \%$ of the study population was found to be fair. Similarly, Johnet al. ${ }^{27}$ stated that $42 \%$ of the subjects, Al Sadhan et al. ${ }^{11}$ reported that $58.2 \%$ of subjects, Tagelsir et al. ${ }^{25}$ specified that $43 \%$ of the subjects had fair oral hygiene status. The main reason for such a poor oral hygiene status would be due to their inability to appreciate deposits on the teeth and visualize the pathological changes brought about by these deposits on the oral tissues leading to neglect during oral hygiene performance. ${ }^{6}$ The guidance and supervision of the caretaker/guardian plays a pivotal role in visualizing, communicating, and aiding in performing acceptable oral hygiene procedures which can turn tides in favor of the visually impaired.

Improper brushing technique and inadequate knowledge about the uses of various oral hygiene aids is also a determinant of oral diseases. The majority of the subjects in the present study used toothbrush (98\%) and toothpaste $(80 \%)$ to clean their teeth. Similarly, in a study by Singh et al., ${ }^{30} 54 \%$ of the subjects used toothbrush and paste on the contrary; Solanki et al. ${ }^{31}$ reported $74 \%$ of the subjects brushed their teeth with tooth powder. In the present study, $61 \%$ of the subjects used a horizontal scrub method of cleaning the teeth. These findings point toward the deficient oral health knowledge and the appropriate use of oral hygiene aids to maintain optimal oral health. Most of the subjects (74\%) in the present study brushed once a day. Similarly, John et al. ${ }^{27}$ in their study stated that $68.6 \%$ of subjects brushed at least once a day. This clearly illustrates that the visually impaired are quite capable of achieving adequate oral hygiene when they communicate with the caregivers and perform oral hygiene measures under their supervision. This hypothesis has been proven effective by Jain et al. ${ }^{22}$ who report oral hygiene of the visually impaired can be comparable to their normal counterparts if the caregivers enforce an oral hygiene routine providing consistent supervision.

\section{Traumatic Dental Injuries}

In our study, the visually impaired group had more occurrences of TDI at $27.6 \%$. The prevalence of TDI according to earlier studies ranges from as low as; Suresan et al. ${ }^{24}(4.62 \%)$ to as high as $50.6 \%$ reported by Singh et al. ${ }^{30}$ Such high prevalence can be due to the lack of preventive strategies in the school environment. Primary preventive measures would be to adopt a safe school environment like; leveled floor lined with an impact-absorbing surface, provision of guide-rails to aid in commuting from one place to the other, removal of obstacles like support columns and walls were not necessary, early and prompt treatment of dentofacial anomalies (over-jet $>9 \mathrm{~mm}$, inadequate lip closure) and provision of assistance for subjects with multiple disorders.

In the present study, the permanent maxillary central incisor was the susceptible tooth to trauma. This observation was similar to the findings of a study completed on disabled subjects. ${ }^{32}$ Permanent maxillary central incisors are more prone to trauma owing to its susceptible and anterior location in the dental arch, an increased protrusion causing lip incompetency, and the anatomy of the teeth which is wide mesiodistally when compared to the labiopalatally exposing greater surface area at trauma risk. Even 
though most of TDI were categorized as mild, none of the subjects had approached oral healthcare personal.

\section{Comprehensive Dental Care Protocol}

Improvements in the oral health status of populations with special needs can be achieved starting with programs that consider the specific protection, health promotion, and curative practices. The CDCP implementation catered to this population's dental service needs. The CDCP contributed to the improvement of the oral health condition of this vulnerable population also providing a platform to dispense oral health education programs utilizing the oral healthcare manpower of an academic dental institution.

\section{Limitations}

There were a few limitations to the present study; the oral health knowledge of the caretaker was not assessed which; could have given us insights into the adequacy of oral hygiene performance by these subjects. Another important drawback of this study was the variability in the sugar consumption history, diet patterns, and oral hygiene practices in different institutions which would affect the generalizability of the study outcomes. Many factors, such as, parent/caretaker education and training in rendering care were not considered.

\section{Recommendations}

At the heart of improving the oral health conditions of this special group lies, health education interventions with a larger emphasis placed on training their parents/caretakers. The dearth of information and educational material readable by the visually impaired in providing knowledge about oral health maintenance was also obvious. Visually impaired subjects should be encouraged to use auditory and tactile media sources for information. Lobby efforts must be made to prioritize oral health among such populations by the Ministry of Social Security and Empowerment of Persons with Disabilities and coordinate efforts between social services and oral healthcare providers.

\section{Conclusion}

Within the limitations of this study, visually impaired subjects showed a higher dental caries experience, a fair oral hygiene status, and extensive TDI. Furthermore, this study population lacked adequate knowledge concerning the appropriate usage of oral hygiene aids, accurate brushing techniques, ill-effects of cariogenic diet, and lacked periodic dental visits. The CDCP was effective to improve access, resolve emergencies, provision of services, and oral health promotion among such neglected populations to bring down the oral health disparity.

\section{References}

1. World Health Organisation. World Report on Vision. Vol 214; 2019. Available from: https://www.who.int/publications/i/item/worldreport-on-vision [Last accessed on 2020 August 02].

2. Gifford KL, Richdale K, Kang P, et al. IMI - Clinical management guidelines report. Investig Ophthalmol Vis Sci 2019;60(3):M184M203. DOI: 10.1167/iovs.18-25977.

3. World Health Organization. Visual Impairment and Blindness (Fact Sheet 282); 011. Available from: http://www.who.int/mediacentre/ factsheets/fs282/en. [Last accessed on 2019 July 24].

4. National Programme for Control of Blindness \&Visual Impairment, Services DG of H, Ministry of Health \& Family Welfare G of I. National Blindness and Visual Impairment Survey India.; 2019. Available from:
https://npcbvi.gov.in/writeReadData/mainlinkFile/File341.pdf [Last accessed on 2020 July 4].

5. Watt RG, Listl S, Peres M, et al. Social inequalities in oral health from evidence to action. Available from http://media.news.health.ufl.edu/ misc/cod-oralhealth/docs/posts_frontpage/Sociallnequalities.pdf [Last accessed on 2020 August 4].

6. Sanjay V, Shetty SM, Shetty RG, et al. Dental health status among sensory impaired and blind institutionalized children aged 6 to 20 years. J Int Oral Health 2014;6(1):55-58.

7. Grace E, Kelmendi M, Fusha E. Oral health status of children with disability living in Albania. Mater Sociomed 2014;26(6):392-394. DOI: 10.5455/msm.2014.26.392-394.

8. Rao D, Amitha H, Munshi AK. Oral hygiene status of disabled children and adolescents attending special schools of South Canara, India. Hong Kong Dent J 2005;2(2):107-112.

9. Reddy K, Sharma A. Prevalence of oral health status in visually impaired children. J Indian Soc Pedod Prev Dent 2011;29(1):25-27. DOI: 10.4103/0970-4388.79922.

10. Schembri A, Fiske J. The implications of visual impairment in an elderly population in recognizing oral disease and maintaining oral health. Spec Care Dentist 2001;21(6):222-226. DOI: 10.1111/j.1754-4505.2001. tb00258.x.

11. Al Sadhan SA, Al-Jobair AM, Bafaqeeh M, et al. Dental and medical health status and oral health knowledge among visually impaired and sighted female schoolchildren in Riyadh: a comparative study. BMC Oral Health 2017;17(1):154. DOI: 10.1186/s12903-017-0446-6.

12. Prashanth ST, Bhatnagar S, Das UM, et al. Oral health knowledge, practice, oral hygiene status, and dental caries prevalence among visually impaired children in Bangalore. J Indian Soc Pedod Prev Dent 2011;29(2):102-105. DOI: 10.4103/0970-4388.84680.

13. Hennequin $M$, Faulks $D$, Roux $O$. Accuracy of estimation of dental treatment needs in special care patients. J Dent 2000;28(2):131-136. DOI: 10.1016/S0300-5712(99)00052-4.

14. Reddy VK, Chaurasia K, Bhambal A, et al. A comparison of oral hygiene status and dental caries experience among institutionalized visually impaired and hearing impaired children of age between 7 and 17 years in central India. J Indian Soc Pedod Prev Dent 2013;31(3):141-145. DOI: 10.4103/0970-4388.117963.

15. Knutson JW, Klein H, Palmer CE. Studies on dental caries. VIII. Relative incidence of caries in the different permanent teeth. J Am Dent Associat Dent Cos 1938;25(12):1923-1934. DOI: 10.14219/jada. archive.1938.0342.

16. Greene JC, Vermillion JR. The simplified oral hygiene index. J Am Dent Assoc 1964;68(1):7-13. DOI: 10.14219/jada.archive.1964.0034.

17. Andreasen JO, Andreasen FM. Classification, etiology and epidemiology. In: Textbook and colour atlas of traumatic injuries to the teeth. 4th ed., Copenhagen: Blackwell Munksgaard; 2011. pp. 218-219.

18. Cavalcanti YW, de Fátima Dantas de Almeida L, de Souza Barbosa A, et al. Planning oral health and clinical discharge in primary care: The comprehensive dental care protocol outcome. J Contemp Dent Pract 2015;16(3):172-177. DOI: 10.5005/jp-journals-10024-1656.

19. Nangia V, Jonas JB, Gupta R, et al. Visual impairment and blindness in rural central India: the central India eye and medical study. Acta Ophthalmol 2013;91(5):483-486. DOI: 10.1111/j.17553768.2012.02447.x.

20. Chang $C$, Shih Y. Knowledge of dental health and oral hygiene practices of Taiwanese visually impaired and sighted students. J Vis Impair Blind 2004;98(5):289-303. DOI: 10.1177/0145482X0409800504.

21. Ozdemir-Ozenen D, Sungurtekin E, Cildir S, et al. A comparison of the oral health status of children who are blind and children who are sighted in Istanbul. J Vis Impair Blind 2012;106(6):362-367. DOI: $10.1177 / 0145482 \times 1210600606$.

22. Jain A, Gupta J, Aggarwal V, et al. To evaluate the comparative status of oral health practices, oral hygiene and periodontal status amongst visually impaired and sighted students. Spec Care Dent 2013;33(2):78-84. DOI: 10.1111/j.1754-4505.2012.00296.x. 
23. Ishtiaq $\mathrm{R}, \mathrm{Chaudhary} \mathrm{MH}$, Rana MA, et al. Psychosocial implications of blindness and low vision in students of a school for children with blindness. Pak J Med Sci 2016;32(2):431-434. DOI: 10.12669/ pjms.322.8737.

24. Suresan V, Das D, Jnaneswar A, et al. Assessment of dental caries, oral hygiene status, traumatic dental injuries and provision of basic oral health care among visually impaired children of Eastern Odisha. J Indian Soc Pedod Prev Dent 2017;35(4):284-290. DOI: 10.4103/JISPPD. JISPPD_48_17.

25. Tagelsir A, Khogli AE, Nurelhuda NM. Oral health of visually impaired schoolchildren in Khartoum State, Sudan. BMC Oral Health 2013;13(1):33. DOI: 10.1186/1472-6831-13-33.

26. Singh A, Dhawan P, Gaurav V, et al. Assessment of oral health-related quality of life in 9-15 year old children with visual impairment in Uttarakhand. India Dental Research Journal 2017;14(1):43-49. DOI: 10.4103/1735-3327.201132.

27. John JR, Daniel B, Paneerselvam D, et al. Prevalence of dental caries, oral hygiene knowledge, status, and practices among visually impaired individuals in Chennai, Tamil Nadu. Int J Dent 2017. 9419648 DOI: $10.1155 / 2017 / 9419648$.

28. Bekiroglu N, Acar N, Kargul B. Caries experience and oral hygiene status of a group of visually impaired children in Istanbul, Turkey. Oral Health Prev Dent 2012;10(1):75-80.

29. Shetty V, Hegde AM, Bhandary S, et al. Oral health status of the visually impaired children--a south Indian study. J Clin Pediatr Dent 2010;34(3):213-216. DOI: 10.17796/jcpd.34.3.j4781g2w8891848u.

30. Singh A, Kumar A, Berwal V, et al. Comparative study of oral hygiene status in blind and deaf children of Rajasthan. J Adv Med Dent Sci Res 2014;2(1):26-31.

31. Solanki J, Gupta S, Chand S. Comparison of dental caries and oral hygiene status among blind school children and normal children Jodhpur City Rajasthan, India. Univers Res J Dent 2014;4(1):22. DOI: 10.4103/2249-9725.127066.

32. Shyama M, al-Mutawa SA, Honkala S. Malocclusions and traumatic injuries in disabled schoolchildren and adolescents in Kuwait. Spec Care Dentist 2001;21(3):104-108. DOI: 10.1111/j.1754-4505.2001. tb00235.x. 